\title{
Sotto il sole giaguaro di Italo Calvino: viaggio attraverso le sensazioni a braccetto con la retorica
}

Le sensazioni e la retorica, la dimensione della percezione e quella delle parole: ecco due mondi opposti, in continua tensione, ma che si attirano come calamite. Tale enigmatico fenomeno di opposizione-attrazione ha affascinato Italo Calvino fin dalle sue prime opere, portandolo a giostrare la sua abile penna tra la realtà concreta delle cose, la spontaneità delle immagini, l'astrazione del pensiero e l'impalpabilità del linguaggio; tutto ciò, nel tentativo di creare una letteratura che sappia rappresentare con armonia questi elementi contrastanti. Con il suo romanzo postumo Sotto il sole giaguaro, Calvino accompagna, per l'ennesima volta, il suo fedele lettore in un viaggio di scoperte e rivelazioni: intrepida, la sua scrittura accoglie la sfida e cerca di descrivere la percezione sensoriale della realtà.

Per capire cosa si cela dietro a tale difficile impresa, facciamo un passo indietro nella produzione calviniana e rileggiamo le prime righe di Se una notte d'inverno un viaggiatore:

Stai per cominciare a leggere il nuovo romanzo Se una notte d'inverno un viaggiatore di Italo Calvino. Rilassati. Raccogliti. Allontana da te ogni altro pensiero. Lascia che il mondo che ti circonda sfumi nell'indistinto. (3)

Ecco che, in quanto lettori, ci troviamo chiamati in causa dal testo stesso che ci accingiamo a leggere, come se la nostra realtà si ritrovasse di colpo immersa nella dimensione esistenziale del romanzo. Si tratta di una sostituzione propriamente metaforica; in questo caso produciamo un senso nuovo grazie al trasferimento del significato di un elemento assente dal testo, ma conosciuto dal lettore-riferimento alla vita, - ad un elemento presente testualmente, ma ancora sconosciuto - la vita nel (e del) testo stesso. Questo trasporto di senso, che conduce alla comprensione, si attua come processo interpretativo bidimensionale. Il lettore deve in parte utilizzare la sua intuizione ed in parte la sua capacità di concettualizzare per poter trarre senso dal testo. ${ }^{1}$ Egli, cioè, oscilla continuamente tra $\mathrm{i}$ due termini che costituiscono la metafora in questione, cioè passa dal testo al mondo, dal mondo al testo. Si sovrappongono così la realtà quotidianamente conosciuta con quella presentata dal sistema narrativo. Il mondo interno prodotto dalla letteratura s'intreccia con il mondo esterno che il testo presenta. In questo modo il linguaggio denotativo, che riproduce la realtà, lascia spazio a quello metaforico, che crea una realtà. Studiando i meccanismi di produzione e di comprensione della metafora, Fonzi e Sancipriano sottolineano che 
la funzione fondamentale del linguaggio metaforico è quella di conciliare l'interiorità con l'esteriorità, il soggettivo con l'oggettivo e di far sì che tale conciliazione sia comunicabile ... II linguaggio denotativo ha la funzione di rispecchiare la realtà, mentre il linguaggio metaforico ha la funzione di creare una nuova realtà . . . Esso è più limitato in quanto non garantisce, come quello denotativo, la comprensione .. . Tuttavia il linguaggio metaforico è più esteso di quello denotativo proprio perché ha in sé quella potenzialità creativa che non impone limiti all'accostamento di elementi, alla creazione di nuovi significati. Rispecchiare la realtà significa infatti essere condizionati da essa, creare la realtà significa padroneggiarla. (15-16)

I concetti di mimesis e poiesis acquistano in questa prospettiva un nuovo valore ed entrano in un rapporto di complementarità. Se da un lato la mimesi come imitazione della realtà si oppone alla poiesi, dall'altro anche l'atto di riprodurre è un'operazione creativa di tipo conoscitivo. Ne è agente il lettore che vuole capire il processo artistico e la rappresentazione del mondo che da esso risulta. Si tratta di azionare un trasferimento metaforico con finalità euristica, cioè di proiettare per intuizione e concettualizzazione il senso del conosciuto sull'ignoto. ${ }^{2}$ Tale processo può avvenire se consideriamo la finzione narrativa in complicità invece che in opposizione con la realtà.

Riprendendo i concetti aristotelici di mythos (storia), mimesis (imitazione del mondo in atto) e del loro rapporto, Singer sottolinea che: I) il testo di narrativa, per essere tale, necessita della mediazione di una realtà, dunque della mimesi come supporto per la storia; II) la rappresentazione è un'espressione linguistica che si organizza strutturalmente per permettere la riflessione; III) la metafora, in senso esteso, è lo strumento retorico utilizzato per collegare il referente reale con quello testuale. La metafora produce un'interazione dinamica che trasferisce il senso dai mondi fittizi a quelli reali (e viceversa), creando così un ponte tra sistemi referenziali diversi $(1-45)$. $^{3}$

La verità e la logica metaforiche escono ovviamente dai parametri del discorso razionale ed oggettivo - discorso d'altronde irrealizzabile in modo assoluto - per fondare una conoscenza intuitiva e percettiva della realtà. Questo tipo di processo cognitivo riconosce che la relazione tra soggetto ed oggetto è complementare e necessaria per definire l'esistenza dell'essere. Scrivono Fonzi e Sancipriano:

Ci sembra quindi di poter individuare un mondo metaforico, diverso dal mondo fisico obbiettivo, che è compito della metafora fare emergere alla coscienza. Se nella realtà obbiettiva l'oggetto è definito, è circoscritto spazialmente e temporalmente, nella realtà metaforica l'oggetto partecipa delle caratteristiche di altri oggetti, è sincreticamente fuso con il contesto, ha un significato labile che varia a seconda della tonalità affettiva del soggetto. Il mondo metaforico è quindi essenzialmente un mondo di partecipazione in cui il soggetto e l'oggetto sono indifferenziati; esso sta alla base di quella conciliazione creativa c consapevole dei due poli che si verifica nella metafora. (5-6)

Lo sconvolgimento dei livelli referenziali e la forza creativa e conoscitiva accordata alla metafora (tematica tipicamente post-moderna) ci ripropongono 
quesiti ontologici ed epistemologici. Chi siamo in rapporto al testo? Qual è la realtà? Come riconoscerla? Quale tipo di forma di conoscenza adottare? Eccoci nell'impasse contro la quale la teoria critica della decostruzione si trova impotente. Siamo cioè al centro della dicotomia tradizionale tra conoscenza oggettiva della realtà esterna e conoscenza condizionata soggettivamente. Se da un lato l'Illuminismo dissacra il valore del mito grazie alla potenza della ragione, dall'altro esso fornisce i mezzi stessi per spingersi oltre alla fede nella razionalità. Come produrre la conoscenza oggettiva se soggetto ed oggetto vivono un'interrelazione continua ove apparato cognitivo e percettivo interferiscono costantemente uno con l'altro? La verità assoluta ed universale non esiste; impera dunque un logocentrismo "malato" perché può dire tutto ma mai né il vero né l'assoluto. Soluzione: gettarsi a capofitto in una realtà che altro non è se non un gioco di parole, lasciarsi prendere dalla vertigine del logos e del sovrappiù di senso. Questo gesto propone un discorso molteplice, polisemico, opaco, autocritico, autorappresentativo, circolare. Ovvero un discorso che si afferma come struttura retorica il cui unico potere è di dichiarare apertamente la propria essenza. E l'essere umano? Egli è il "verbo", esiste grazie alla parola attraverso la quale comunica cioè vive in un ambiente sociale, dunque egli è a sua volta oggetto retorico. Ma veramente non c'è più via di uscita dal labirinto del logocentrismo e della significazione sempre procrastinata, poiché indicibile e indefinibile in modo assoluto?

Calvino ci propone un'alternativa con Sotto il sole giaguaro. Egli utilizza la ricchezza significativa dei tropi per suggerire al lettore nuove possibili soluzioni per nominare ed interpretare il mondo ed il testo. La "retorica" calviniana opera con le parole, ma chiama in causa le facoltà percettive che permettono di giocare con la memoria, l'immaginazione, le emozioni, la psiche ed il fisico. Due chiarimenti: I. siamo nell'ambito del testo inteso come metafora di come l'individuo può giungere alla conoscenza della realtà; II. il "modus operandi" in cui la metafora giunge ad un tipo di sapere può fungere da modello cognitivo ed è il metodo dell'interrelazione (combinazione di sensi, concetti, intuizioni, parole). Come scrive Eco:

Non si tratta di dire che esistono anche metafore visive (all'interno dell'universo visivo bisognerà distinguere i sistemi figurativi, quelli gestuali e così via) o che esistono anche-forse-metafore olfattive o musicali. Il problema è che la metafora verbale richiede spesso, per essere in qualche modo spiegata nelle sue origini, il rinvio a esperienze visive, auditive, tattili, olfattive . . . Non ci interessa la metafora come ornamento, perché se fosse solo questo ... essa sarebbe completamente spiegabile nei termini di una teoria della denotazione. Interessa come strumento di conoscenza additiva e non sostitutiva. (Semiotica 143)

Metafora come conoscenza "additiva" che ha il potere di valicare $\mathrm{i}$ limiti delle categorie logiche e delle definizioni denotative. Come? Considerando il testo come messaggio linguistico, possiamo dire con Singer che la metafora è un'attività testuale, ma dinamica, che mescola i meccanismi della produzione di senso: 
Metaphoric meaning arises from a transgression of contextual limits and thereby entails a reconstruction of the discoursive ground from which it springs . . By definition a transference of meaning across categorical boundaries. (23)

Essa implica infatti un processo transfrastico ed intertestuale. I cinque sensi sono alla radice della comprensione; la scrittura e la lettura si inseriscono poi di fianco a loro per registrare la storia e produrre un futuro possibile dell'umanità - potremmo parlare dunque di sette sensi. Ricordiamoci qui di una conosciuta metafora medioevale (si vedano Allan di Lille, Ugo da San Vittore): il mondo è il libro scritto da Dio, dunque per conoscere e capire i fenomeni della realtà bisogna leggere il mondo come un libro. Alla base della tematica calviniana sta quindi un problema prettamente epistemologico affrontato però in termini retorici-discorso della convinzione, della possibilità e della probabilità, arte (techne) del sillogismo, ordine del desiderio-e non in termini filosofici o scientifici-discorso della verità assoluta, della conoscenza oggettiva, della ragione, dell'ordine logico.

Sotto il sole giaguaro, di pubblicazione postuma, raccoglie tre racconti il cui leitmotiv è la percezione della realtà attraverso i sensi. Questa tematica esplicita sussume il problema, tipicamente calviniano, del processo della lettura. L'enfasi messa sulla percezione sensoriale suggerisce al lettore l'uso di tale strumento per attuare la lettura dell'opera. Singer intravede nella narrativa moderna un interesse particolare per i processi di ricezione del testo (l'epifania di Joyce); leggere deve essere un momento di trasformazione: "The mind placing itself again in the turbulent flux of experience" (27). In Sotto il sole giaguaro si tratta di leggere e percepire il testo attraverso i sensi descritti: l'olfatto ne "Il nome, il naso", il gusto in "Sotto il sole giaguaro", l'udito in "Un re in ascolto". I I tre testi si dispiegano su vari assi temporali e spaziali. In quanto racconti, non sono centrati sulla progressione lineare degli eventi e non si svolgono in luce di una catarsi finale, di una risoluzione dei quesiti o di un "dénouement". Frammentati per quanto riguarda la loro struttura narrativa, i tre racconti sono in effetti perfettamente coordinati a livello delle isotopie semantiche, della tematica e del messaggio da interpretare e cioè: la dimostrazione scritta del potere dei sensi che vuole fissarsi nel circuito della comunicazione per raggiungere il pubblico. Grazie a questa strategia narrativa il lettore ha la libertà di scegliere il punto da cui iniziare o terminare il libro; egli è invogliato dalla divisione testuale a designare momenti distinti alla lettura di ogni racconto. In tal modo, il lettore può dedicarsi a suo piacimento allo studio di uno dei sensi, concentrandosi sulle sensazioni risvegliate dalla narrazione ed instaurando così un contatto empatico tra la sua realtà sensoriale ed il testo.

Nel racconto iniziale la narrazione in prima persona conduce il lettore in un andirivieni tra eventi diversi del passato: iniziamo con la decadente Parigi per passare alla savana della preistoria, poi allo spirito ribelle dell'Inghilterra degli "Hippies"; approdiamo, ogni volta che il narratore prende fiato (nel 
testo degli spazi bianchi interrompono il flusso del discorso), ad un tempo ed un luogo diverso. Ciò che conferisce al frammentato racconto unità e coerenza è l'inseguimento di una traccia: il profumo lasciato da una presenza femminile. Questo indescrivibile ed inconfondibile odore passa attraverso gli episodi sviluppati in luoghi e tempi diversi, permea le righe del testo, fino ad impregnare perfino l'olfatto del lettore condotto da questo nell'intreccio del racconto. Profumo: guida verso l'identità di una persona, mezzo di classificazione, punto di orientamento, lessico dell'“alfabeto dell'olfatto" (Sotto 7), strumento della conoscenza, elemento narrativo di coordinazione:

Era appunto questo che io chiedevo alla precisa esperienza di Madame Odile: di dare un nome a una commozione dell'olfatto che non riuscivo né a dimenticare né a trattenere nella memoria senza che sbiadisse lentamente ... In questo andirivieni nella scala degli odori mi perdevo, non sapevo più discernere la direzione in cui inseguire il mio ricordo, sapevo solo che in un punto della gamma s'apriva un vuoto, una piega nascosta dove s'annidava quel profumo che era per me tutta una donna. (Sotto 11)

E non facevamo forse così quando la savana la foresta la palude erano una rete d'odori e correvamo a testa bassa senza perdere il contatto col terreno aiutandoci con le mani e col naso a trovare la strada, e tutto quello che dovevamo capire lo capivamo col naso prima che con gli occhi . . . tutto è nel naso, il mondo è il naso, noi del branco è col naso che sappiamo chi è del branco e chi non è del branco, le femmine del branco hanno un odore che è l'odore del branco, e poi ogni femmina ha un odore che la distingue dalle altre femmine. (Sotto 12)

Così la gente la riconosco dall'odore ... E io mi ricordo che stavo andando a mettere i pennies nella stufa e mi rialzo mi stacco dall'isola del suo odore continuo la mia traversata in mezzo ai corpi sconosciuti in mezzo a odori incompatibili anzi repulsivi, cerco . . . nel buio nel chiuso nel fiato dei corpi, adesso devo attraversare di nuovo per ritrovare quella ragazza di cui non so che l'odore, è difficile cercare nel buio ma anche se la vedessi come faccio per sapere se è lei non so altro che l'odore. (Sotto 15-17)

Quando i profumi si mescolano e la traccia si perde, allora il frammentato racconto riconduce le tre dimensioni narrative dagli stili diversi-Parigi, stile ricercato e decadente; savana primitiva e Inghilterra "hippy", linguaggio duro, diretto, dal ritmo serrato e veloce, dalla punteggiatura ridotta al minimo - ad un'unica tematica finale: il riconoscimento della morte. Nella morte la figura femminile perde il suo odore e la sua identità; resta solo l'eco del suo sentore in mezzo al miscuglio disgustoso di altri odori, altre identità, rimane una tenue scia associata oramai ad immagini di decadimento, al nome della morte:

Già nell'atrio mi raggiunge un odore di fiori pesante, come di vegetazione imputridita; ... ma riconosco il fondo, l'eco del profumo che non somiglia a nessun altro, oramai fuso con l'odore di morte come se fossero stati inseparabili da sempre. 
E quell'odore che seguivo è laggiù che si è perso, è laggiù che a seconda di come tira il vento viene fuori insieme al fetore di cadaveri sbranati al fiato degli sciacalli che li sbranano ancora caldi al sangue che si asciuga sulle rocce al sole.

E a tirarla fuori per le sue gambe rigide sento il suo odore dentro quell'odore asfissiante, il suo odore che cerco d'inseguire e di distinguere nell'ambulanza nel pronto soccorso negli odori di disinfettanti e di liquame che scola dai tavoli di marmo dell'obitorio e l'aria ne resta impregnata specialmente quando fuori il tempo è umido. (Sotto 22-24)

Grazie ad un'utilizzazione retorica della scrittura-parola tecnicamente destreggiata, atta a creare le condizioni per commuovere, trascinare, far sentire al lettore il concreto, "l'odore" delle immagini-Calvino è in grado di far percepire e riconoscere dal suo lettore la potenza del senso dell'olfatto. Come suggerito dal titolo del racconto, "Il nome, il naso", il valore dell'odore sta nel poter nominare con sicurezza, senza errori e senza ambiguità l'oggetto del desiderio, cioè l'oggetto da conoscere che abita la nostra realtà. Nel momento in cui quest'oggetto viene a mancare, anche il suo profumo svanisce ed il fatto di riconoscerlo e di nominarlo non ha più importanza.

Nel suo cammino attraverso la storia, Calvino si sofferma su tre momenti distinti eppur collegati del viaggio dell'umanità verso la conoscenza. Perché questa scelta? Perché la preistoria, l'inizio del nostro secolo, gli anni sessanta furono tre periodi in cui l'individuo si rivolse spontaneamente alla percezione sensoriale per conoscere la sua realtà; nel primo momento si trattò di necessità, in quelli seguenti di un ritorno nostalgico e ribelle all'euforia dei sensi, alla libertà di affermare il proprio modo individuale di sapere. Nella preistoria l'uomo si spinge dalle sue quattro gambe verso l'alto, inizia a camminare eretto ed a scoprire nuovi modi di capire il suo mondo, cambia la sua prospettiva sulle cose, vuole capire l'insieme non solo il dettaglio, vuole vedere lontano. Il naso non gli basta più, ha bisogno di altri sensi:

Ci sono certi tra di noi che hanno preso l'abitudine di camminare senza posare mai lc mani per terra ... A tencre il naso quassù sospeso nell'aria certo le cose che si perdono sono tante: notizie che puoi tirar fuori annusando la terra con tutte le tracce di bestie che ci sono passate, annusando gli altri del branco specialmente le femmine. Ma si hanno in cambio altre cose: il naso più asciutto che sente odori lontani portati dal vento $i$ frutti degli alberi le uova degli uccelli nei nidi. E gli occhi aiutano il naso, afferrano ncllo spazio le cose, le foglie del sicomoro, il fiume, la striscia azzurra della forcsta, le nuvolc. (Sotto 20-21)

Nella descrizione di quest'essere primitivo, per la prima volta tentennante su due gambe, si racchiude metaforicamente l'evoluzione della conoscenza. Come l'individuo preistorico non si accontenta dell'olfatto e si rivolge ai cinque sensi per conoscere ciò che lo circonda, così quello del ventesimo secolo non accetta più di conferire esclusivamente al linguaggio tutto il potere di cognizione e trasmissione della realtà. Calvino dunque propone una 
conciliazione tra sensi e parola; egli utilizza la scrittura-grafema delle sue parole - per rieducare il lettore all'uso dei sensi, alla lettura delle sensazioni, alla conciliazione dell'intuizione, della percezione e della logica. Imparando la lezione, possiamo congetturare la nostra "lettura" come un insieme di relazioni a più livelli delle nostre facoltà intellettuali e percettive. Iniziamo dalla copertina stessa del testo ove vediamo rappresentati dei segni multicolori, organizzati in sequenze, strutturati secondo un ordine sintagmatico (orizzontalità) e paradigmatico (verticalità). Si tratta di un particolare dell'opera di Vasilij Kandinskij, Successione. Come i tre testi seguenti, anche questo è un frammento, un racconto estratto dall'insieme del quadro, una serie di geroglifici, forma di scrittura, forma di comunicazione, forma di rappresentazione e di conoscenza. Questi segni colpiscono i nostri sensi di lettori e producono una sensazione, un senso, causato da più fattori: dai loro colori, dalla loro successione, dalle associazioni mentali della nostra psiche, dalla vicinanza con il testo scritto che segue. Iniziamo finalmente la lettura dei racconti avendo già uno schema mentale ove sono registrate queste prime impressioni che inevitabilmente colleghiamo al testo. Non è solo la parola che può conferire un significato al discorso letterario; anche la conoscenza noetica e quella estetica possono produrre senso. In Sotto il sole giaguaro è forse proprio questo tipo di sapere a conferire significato al testo. Si tratta di una conoscenza intesa come percezione di apparenze e di fenomeni, come registrazione di sensazioni che passano dall'atto della lettura a quello della costruzione mentale attraverso le intuizioni e le associazioni.

Ecco che nel secondo racconto, dal titolo omonimo al libro, ${ }^{5}$ la decodificazione della realtà avviene nuovamente tramite i sensi - la vista, il gusto - ed è descritta dalla parola. La voce narrante, nuovamente maschile, ci porta in Messico ed è a tratti accompagnata da quella di una donna. Siamo in una dimensione presente, immersi nei profumi seducenti della natura solare e nei piccanti sapori della cucina messicana, ma ci troviamo anche nel misterioso passato dei polverosi ed assolati templi aztechi. Il racconto si apre con un'immagine di un quadro che il narratore e la sua compagna studiano attentamente, mettendo in risalto, fin dall'inizio, la complicità testo-quadroparola-sensazioni:

La prima cosa che avevamo notato era un quadro . . . Il quadro era una grande tela oscura che rappresentava una giovane monaca e un vecchio prete, in piedi, affiancati, le mani leggermente staccate dal corpo, quasi sfiorandosi ... La parte inferiore del quadro era occupata da una lunga didascalia, in fitte righe d'una angolosa scrittura corsiva, bianco su nero . . L La ragione per cui venivano ritratti insieme era lo straordinario amore (la parola nella pia prosa spagnola si presentava carica del suo anelito ultraterreno) che aveva legato per trent'anni la badessa e il suo confessore, un così grande amore (la parola amore nella sua accezione spirituale sublimava ma non cancellava l'emozione corporea) che quando il prete era venuto a morte, la badessa, di vent'anni più giovane, nello spazio di un giorno, s'era ammalata ed era spirata letteralmente d'amore (la parola bruciava d'una verità in cui tutti i significati convergono) 
per raggiungerlo in cielo.

Olivia, che sapeva lo spagnolo meglio di me, m'aiutò a decifrare la storia . . . ; e furono queste le sole parole che ci venne da scambiare durante e dopo la lettura, come ci trovassimo in presenza d'un dramma . . . Così cerco di descrivere qucl che provavo io: il senso d'una mancanza, d'un vuoto divorante. (Sotto 29-30) [enfasi mia]

Iniziamo anche qui (come noi lettori del libro iniziamo dal frammento di Kandinskij) da un quadro da decifrare, ma il narratore si dichiara insoddisfatto di fronte alle sue parole descrittive. In breve, ci rendiamo conto che tutto il racconto è un esercizio per segnalare con il linguaggio quello che solo le sensazioni sanno tradurre: il rapporto erotico tra narratore-donna-cibo-sapere. Poiché si tratta di un testo scritto, il render presente e il far sentire al lettore questa relazione richiede l'uso di figure retoriche (metafora, similitudine, metonimia, sineddoche, sinestesia) che agiscono direttamente sull'emotività, l'intuizione e l'immaginazione del lettore. La percezione della realtà testuale deve avvenire "concretamente" come se si trattasse della realtà esterna al racconto. Per esempio, ecco alcuni momenti in cui Calvino riesce a conseguire tale effetto:

L'idea delle monache evocava in noi i sapori di una cucina elaborata e audace, come tesa a far vibrare le note estreme dei sapori e ad accostarle in modulazioni, accordi e soprattutto dissonanze che s'imponessero come un'esperienza senza confronti, un punto di non ritorno, una possessione assoluta esercitata sulla ricettività di tutti i sensi. (Sotto 31)

Da una località all'altra il lessico gastronomico variava proponendo scmpre nuovi termini da registrare e nuove sensazioni da distinguere. (Sotto 34)

In realtà invece il desiderio che tutta la sua persona esprimeva era quello di comunicarmi ciò che sentiva: di comunicare con me attraverso i sapori, o di comunicare coi sapori attraverso un doppio corredo di papille, il suo e il mio. (Sotto 35-36)

Era la sensazione dei suoi denti nella mia carne che stavo immaginando, c sentivo la sua lingua sollevarmi contro la volta del palato, avvolgermi di saliva, poi spingermi sotto la punta dei canini. (Sotto 51)

Come nel primo racconto, anche qui ritorna il tema della morte come fine della percezione e della necessità di conoscere. Solo che ora si tratta della vita che continua come fosse già la morte, poiché privata delle sensazioni: "Forse la morte del tempo riguarda solo noi', rispose Oliva, 'noi che ci sbraniamo facendo finta di non saperlo, facendo finta di non sentire più i sapori ..." (Sotto 49). Calvino insiste dunque sul valore intrinseco della percezione come strumento fisiologico per conoscere la realtà e soprattutto come attributo specifico dell'essere umano. Privo di sensazioni l'individuo 
è inesistente, non comunica né all'altro né a sé stesso. Sensazioni e parola si completano in una logica della vita che non deve essere necessariamente quella della ragione e della verità assolute ed oggettive. Anche l'intuizione e la percezione conducono ad un sapere: "Il nome, il naso", "Sapore, sapere", ci dice Calvino (Fonzi e Sancipriano 5-6).

Con "Un re in ascolto" siamo kafkianamente prigionieri, lettori e protagonista, di un palazzo di cui è descritto il labirintico interno, il sottosuolo pieno di cunicoli misteriosi e la circostante città. La rappresentazione di tali luoghi non passa per la vista, ma per l'udito: è l'orecchio che vede. Il protagonista è un re bloccato fisicamente nel suo ruolo; l'autorità gli è conferita esclusivamente dalla sua posizione precaria sul trono:

Del resto, che bisogno avresti di darti tanto da fare? Sei re, tutto quello che desideri è già tuo ... Insomma, tutto è stato predisposto per evitarti qualsiasi spostamento. Non avresti nulla da guadagnare, a muoverti, e tutto da perdere. Se t'alzi, se t'allontani anche di pochi passi, se perdi di vista il trono anche per un attimo, chi ti garantisce che quando torni non ci trovi qualcun altro seduto sopra? Magari uno che ti somiglia, uguale identico. Va' poi a dimostrare che il re sei tu e non lui! Un re si distingue dal fatto che siede sul trono, che porta la corona e lo scettro. Ora che questi attributi sono tuoi, meglio non te ne stacchi nemmeno per un istante. (Sotto 62-63)

Lo stile narrativo è coerente all'immagine statuaria del re: non si tratta come nei racconti precedenti di una narrazione di eventi in prima persona, ma di un monologo del narratore che si rivolge al suo narratario. Questi è il re stesso, la seconda persona interrogata ma privata della possibilità di esprimersi direttamente. Poiché bloccato sul trono nella prigione delle sue funzioni politiche, il re vive solo attraverso l'udito che gli permette di sentire e percepire il mondo. Il narratore può dunque descrivere solo i particolari psicologici, i gesti, le sensazioni di questo personaggio-orecchio. Ogni suono prodotto nello spazio del palazzo viene interpretato, decodificato, come un messaggio in alfabeto morse $o$ in codici segreti:

Vicino al trono c'è uno spigolo del muro da cui ogni tanto senti venire una specie di rimbombo: colpi lontani come il bussare a una porta. C'è qualcuno che picchia dall'altra parte? ... Sono segnali? . . Allungando il braccio puoi battere col pugno contro lo spigolo. Ripeti i colpi come li hai uditi ... Hai stabilito un dialogo? Per dialogare dovresti conoscere la lingua ... Sono segni traducibili in un codice? Qualcuno sta formando lettere, parole? (Sotto 74-75)

Tutto il mondo è percepito attraverso i rumori: il tempo è la ripetizione di suoni conosciuti, il contatto con altri umani è il tocco su una parete, la città è un ronzio che viene dalla finestra, la donna è una voce sconosciuta ed incantevole. Si instaura gradualmente una relazione metaforica tra re e palazzo. Si tratta di un rapporto necessario: il re può sentirsi vivere solo attraverso i messaggi che gli invia il suo palazzo. Solo così, cioè facendosi un'immagine del mondo e confrontandosi con l'esistenza dell'“altro", egli acquista un'identità. Assistiamo ad una lenta trasformazione: 
11 palazzo è un orologio. (Sotto 65)

$\mathrm{Nel}$ grande lago del silenzio in cui tu galleggi sfociano fiumi d'aria mossa da vibrazioni intermittenti; tu le intercetti e le decifri, attento, assorto. Il palazzo è tutto volute, tutto lobi, è un grande orecchio in cui anatomia e architettura si scambiano nomi e funzioni: padiglioni, trombe, timpani, chiocciole, labirinti; tu sci appiattito in fondo, nella zona più interna del palazzo-orecchio, del tuo orecchio; il palazzo è l'orecchio del re. (Sotto 66)

Il palazzo è il corpo del re. Il tuo corpo ti manda messaggi misteriosi, che tu accogli con timore, con ansia. (Sotto 71)

Grazie all'udito il re capta e capisce la sua realtà. Questa conoscenza è però di tipo speculativo, intuitivo: è la conoscenza della metafora. Il re percepisce le pulsazioni del mondo circostante e le trasforma nella sua mente in immagini, in oggetti concreti, utilizzando spesso il vocabolario che conosce per definire ciò che non ha ancora presente (vedi nella citazione precedente lo scambio di termini anatomici ed architettonici). Si crea la realtà "immaginando intorno a ogni segno sonoro pareti, soffitti, impiantiti, dando forma al vuoto in cui i rumori si propagano" (Sotto 70). Conoscere vuol dire udire: "Ciò che i tuoi orecchi cercano di apprendere, su di te, sul tuo destino" (Sotto 68). Desiderare vuol dire udire: "T' attrae quella voce in quanto voce, come si offre nel canto" (Sotto 82). Vedere vuol dire udire: "La voce di donna che ti chiama e la tua voce che la chiama devi captarle insieme nella stessa intenzione d'ascolto (o vuoi chiamarlo sguardo dell'orecchio?)" (Sotto 84-85).

Il re mantiene la sua identità fintantoché resta concentrato con l'udito sul suo palazzo che gli invia ripetutamente gli stessi segnali, ma il desideriocome negli altri racconti è la donna, la voce di donna, la voce di un'altra vita - disturba la comunicazione. I suoni vengono ora dalla città, dall'esterno ignoto, da un mondo che il re non possiede e dunque non esiste: " $E$ inutile cercare te stesso in un mondo che non t'appartiene, che forse non esiste" (Sotto 87). Ma oramai il "rumore" si c̀ inserito nei messaggi, i rumori si mescolano e non sono più identificabili, un'esplosione invade lo spazio e paradossalmente il fragore del caos crea il silenzio. Ora è necessario rivolgersi ad altri mezzi per conoscere, ad altri sensi. Il re si desta come da un incubo e si guarda intorno, sente il vento sul viso, ode i rumori della città che si risveglia. Finalmente, all'esterno del palazzo e della prigionia del potere, il re è libero dal giogo delle congetture mentali impostegli dall'unico mezzo di conoscenza disponibile: il suo udito. La vita è questione di sinestesia, di associazioni d'immagini, di percezioni accostate, di trasposizioni di senso e sensazioni, di metafore continue. ${ }^{6}$

Procedendo nella lettura dei tre racconti, percepiamo dunque con un certo disagio - presto si trasformerà in infatuazione, eccitazione, "jouissance" - che 
il linguaggio ed i suoi alfabeti non sono gli unici mezzi che ci comunicano un messaggio, benché ci troviamo all'interno del reame della scrittura. Il testo stesso denuncia con innovative analogie tra scrittura, parola e sensazioni la debolezza della parola denotativa, oggettiva, considerata solo come strumento di una descrizione logica e razionale della realtà, priva di creatività e delle congetture fantastiche ed intuitive proprie all'umanità. Scrive Calvino con tono nostalgico:

Come epigrafi in un alfabeto indecifrabile, di cui metà delle lettere siano state cancellate dallo smeriglio del vento carico di sabbia, così voi resterete, profumerie, per l'uomo futuro senza naso ... Dimenticato l'alfabeto dell'olfatto che ne faceva altrettanti vocaboli, d'un lessico prezioso, i profumi resteranno senza parola, inarticolati, illeggibili. (Sotto 7-8)

Ma come potevo io descrivere a parole la sensazione languida e feroce che avevo provato la sera prima al ballo mascherato quando la mia misteriosa compagna di valzer con un gesto pigro aveva fatto scorrere lo scialle di velo che separava la sua bianca spalla dai mici baffi e una nuvola striata e flessuosa m'aveva aggredito le narici come se stessi aspirando l'anima d'una tigre? (Sotto 10)

Non ci sono parole né notizic più precise di quelle che riceve il naso. (Sotto 12)

Calvino vuole andare oltre all'impasse del logocentrismo, del regno della parola assoluta; perciò si rivolge ad un passato in cui i sensi comandano la comunicazione e la conoscenza. Solo la complicità dei sensi e del linguaggio può liberarci da questo vicolo cieco, così come per il narratore ed Olivia solo l'intenzione di percepire la loro vita coinvolgendo passato e presente, mistero e realtà, soprattutto gusto ed erotismo, può condurre alla comunicazione:

Questo bisogno che aveva Olivia di coinvolgermi nelle sue emozioni . . mi dimostrava quanto le fossi indispensabile e come per lei i piaceri dell'esistenza fossero apprezzabili solo se condivisi tra noi ... E non potevo notare che certe manifestazioni della carica vitale d'Olivia . . continuassero a dispiegarsi sotto i miei occhi senz'aver perso nulla della loro intensità, con una sola variante di rilievo: l'aver per teatro non più il letto dei nostri abbracci ma una tavola apparecchiata. (Sotto 36-37)

Per rendere "leggibile" la percezione dell'immagine visiva, Calvino investe i tropi di un insolito valore euristico. Soprattutto le metafore e le analogie riescono nella narrazione calviniana a concretizzare l'immagine di una realtà conosciuta-intuita-attraverso i sensi. L'atto di narrare, e conseguentemente di leggere, passa in secondo piano per mettere in risalto il significato "fenomenologico" dell'opera: la realtà si può solo descrivere con le parole se vogliamo comunicarla, ma per conoscerla e dominarla possiamo, anzi dobbiamo prima percepirla e sentirla. Come fornire attraverso la scrittura la concretezza delle sensazioni, lo sconvolgimento che provoca il contatto concreto con il mondo? 
Chiamato in gioco da Calvino è il valore euristico delle immagini che produce la metafora: i racconti sono metafore e come tali producono senso e lo attribuiscono alla realtà. I tropi non sono portatori di significato, ma indicatori di direzioni da seguire (dal greco tropos, cioè direzione) per giungere al senso spesso suggerito dalla metafora (dal greco metapherein, cioè trasferire). I] testo si presenta al lettore come una possibile via cognitiva la cui chiave interpretativa ci è data dalla sua stessa composizione narrativa e retorica: $\mathrm{i}$ racconti sono indicatori di direzione e trasferitori di senso. Perché raccontometafora? Poiché essi sono jl movimento, sia narrativo che esistenziale, verso la conoscenza fornita dalla percezione individuale di quel fenomeno che è la realtà. Beninteso, non una realtà assoluta ed oggettiva, ma una realtà possibile proprio perché individuale, multiforme, moltiplicata dalla percezione che la raggiunge. In questa prospettiva il mondo del racconto non è descrivibile obiettivamente; esso acquista significato solo se visto in relazione con quello singolare proprio ad ogni lettore. Il senso è dato da ciò a cui la narrazione allude, ciò che non è rappresentato e non è nominato. Si tratta dunque di un senso fornitoci tramite l'uso della metafora, tropo dell'assenza, dell'allusione e dell'intuizione. Come ricordano Fonzi e Sancipriano:

Tutta la nostra conoscenza è di tipo relazionale . . Poiché la metafora mette in relazione elementi (appartenenti o entrambi alla sfera concreta o uno alla sfera astratta e l'altro a quella concreta), essa rappresenta un modo di conoscere ... Se la metafora è modo di conoscere, essa è tuttavia un tipo di conoscenza non razionale ma intuitivo, potendosi anzi affermare che offre un'alternativa alla conoscenza di tipo logico. Mettendo in rapporto l'interiorità o l'ottica soggettiva con l'esteriorità, la metafora costituisce una conoscenza di tipo intuitivo, fondata sull'immagine e non sulla dimostrazione. $(47-48)$

Con sorridente arguzia e impegno genuino, Calvino usa il linguaggio come arma contro il linguaggio stesso e la letteratura come denuncia dei limiti di quella, ma egli non si ferma al semplice "J'accuse". Il suo passo innovatore sta nel riconoscere da un lato l'impotenza del discorso letterario e dall'altro la sua forza in rapporto alla definizione e conoscenza dell'umanità. Il gioco di Calvino consiste nel presentare mediante il testo scritto il modo in cui l'essere umano svolge, attraverso la sua evoluzione, la matassa della conoscenza. Si tratta di conoscere la realtà attraverso le immagini che l'individuo si può fare di essa. La scrittura è qui pretesto per raccontare-disegnarefar sentire come l'individuo percepisce il mondo che lo circonda. È, nel suo essere narrazione, il modello-al secondo grado (Ricoeur, nota 2)-di quell'ars combinatoria che si pone come base del fenomeno che è la vita. Le vite umane, citiamo Kundera in L'insostenibile leggerezza dell'essere, "sono costruite come una composizione musicale" (59). Infine, è in quanto testo fittizio uno strumento euristico per "afferrare" i processi della produzione di senso che, come semiosi illimitata, non devono necessariamente limitarsi ai rapporti razionali, logici, assolutistici imposti dalla nostra tradizione filosofica 
e scientifica. Il referente dei tre racconti è simile a quello della vita quotidiana (ci permette così la comprensione del testo), ma si distende su un livello metaforico che concede l'esplorazione di nuovi modi di conoscere e produrre il senso poiché spezza "il legame referenziale abituale tra segno e significato, ravvicina elementi che nella realtà sono differenziati, e condensa sincreticamente in una singola immagine due significati" (Fonzi e Sancipriano 19). La dimensione del racconto è il luogo della congettura e della contingenza, del misterioso incontro tra autore e lettore, tra mondo testuale e mondo reale, tra sensazioni e scrittura. Per citare ancora Kundera:

I personaggi del mio romanzo sono le mie proprie possibilità che non si sono realizzate. Per questo voglio bene a tutti allo stesso modo e tutti allo stesso modo mi spaventano: ciascuno di essi ha superato un confine che io ho solo aggirato. È proprio questo confine superato (il confine oltre il quale finisce il mio io) che mi attrae. Al di là di esso incomincia il mistero sul quale il romanzo si interroga. Un romanzo non è una confessione dell'autore, ma un'esplorazione di ciò che è la vita umana nella trappola che il mondo è diventato. (226)

Con Sotto il sole giaguaro siamo nel reame misterioso e ricco di un neobarocco contemporaneo che gioca sull'arte di combinare ed incastrare i pezzi della narrazione (superficie, strutture sintattiche e semantiche, tematiche, immagini, parole, sensazioni, significati). A proposito dell'arte barocca scrive Conte che la costruzione del mondo testuale è un modello del "fare" e del "da farsi", un modo per nominare e dunque dominare la realtà, per comporla e scomporla, per fissarla nella parola, ma allo stesso tempo denunciarne il carattere mutevole, effimero e polisemico (si vedano 11-54). Nel testo barocco e nei racconti di Calvino così lavora la retorica ed in particolare la metafora, "prolungamento dell'assenza del segno virtuale e diramazione laterale del significato del segno presente" (Conte 40). Lo stesso Calvino ci suggerisce tramite un'ardita similitudine di vedere arte e mondo come gioco eseguito a più livelli fenomenologici di incastri e sovrapposizioni di immagini, sensazioni e parole:

Né si poteva dimenticare l'architettura sacra che faceva da sfondo alle vite di quelle religiose, mossa dalla stessa spinta verso l'estremo che portava all'esasperazione dei sapori amplificata dalla vampa dei chiles più piccante. Così come il barocco coloniale non poneva limite alla profusione degli ornamenti e allo sfarzo, per cui la presenza di Dio era identificata in un delirio minuziosamente calcolato di sensazioni eccessive e traboccanti, così il bruciore delle quarantadue varietà indigene di peperoncini sapientemente scelti per ogni vivanda apriva le prospettive d'un'estasi fiammeggiante. (Sotto 33)

I tre racconti che formano il libro si sviluppano secondo un modello retorico, ovvero di utilizzazione tecnica di un discorso organizzato da scopi predeterminati e ordinato secondo le proprie esigenze interne. Si tratta per Calvino di spingere la scrittura al di là di sé stessa poiché il testo, inteso come sistema di segni, riporta un'immagine mentale sulla carta, indica senza 
imporre la denominazione. Il leitmotiv è quello della comunicazione di una conoscenza percettiva. Tale progetto si attua grazie all'uso della retorica intesa qui sia come tropologia (per esempio produzione e comprensione di metafora, similitudine, metonimia, sineddoche, ecc.) che come tecnica per colpire il lettore, cioè implicarlo nell'azione narrativa, attirare la sua attenzione, raggiungere la sua emotività, creare in lui dubbi, suscitare desideri e permettergli di vedere le cose in modo nuovo. Ovviamente, $i$ racconti così costruiti rischiano di non venir percepiti dal lettore privo di una creatività sua, limitato dai canoni tradizionali della letteratura e contento di rimanere imprigionato nella sicurezza del linguaggio denotativo che descrive una realtà già conosciuta. Dobbiamo essere lettori "metaforici":

L'individuo rigido, fissato funzionalmente sugli oggetti, l'individuo che accetta la realtà come dato di fatto, non può essere metaforico. L'individuo metaforico concepisce invece la realtà come oggetto di continue ristrutturazioni, la interpreta in funzione della sua dinamica affettiva, è disposto ad entrare in conflitto con essa e a risolvere tale conflitto creando nuove aree di esperienza ... Il pensiero è secondo noi metaforico nella misura in cui postula ipotesi, crea modelli e teoric attraverso la trasposizione di dati dell'esperienza. (Fonzi e Sancipriano 7)

Ecco dunque che ci disponiamo come tali di fronte alla lettura di Sotto il sole giaguaro e del mondo. I messaggi lasciati dalla scrittura non solo sono decodificati attraverso un processo grammaticale e logico, ma anche grazie ad un "reading" (P. De Man) dei messaggi stessi attraverso un processo retorico che implica la complicità di tutti i sensi con la parola ed il meccanismo interpretativo. Si tratta cioè di un'interpretazione continua che da un segno conduce ad un altro, anche di natura diversa, e così via. ${ }^{7} \mathrm{Da}$ parola scritta a immagine mentale a percezione a sensazione, da un racconto all'altro attraverso l'olfatto, il gusto, l'udito e la parola, in un moto perpetuo.

\section{Université de Montréal}

\section{NOTE}

1 Scrivono Fonzi e Sancipriano che per produrre e comprenderc la metafora l'individuo si avvale della sua intuizione e della sua capacità di costruire concetti. "L'intuizione permette all'individuo di cogliere con un atto immediato la somiglianza tra i due elementi, il rapporto cmpatico e sincretico esistente tra di essi; la concettualizzazione implica un processo di astrazione degli elementi comuni . . . Nella metafora l'intuizione e la concettualizzazione sono duc processi interdipendenti. Quando infatti prevale solo il polo intuitivo, senza che questo sia in equilibrio con quello concettuale, la metafora è autistica, non comunicabile ...; quando invece prevale il polo concettuale senza che questo sia in equilibrio con qucllo intuitivo, la metafora che ne risulta perde la sua pregnanza iconica, la sua suggestività ed è quindi una mclafora 'morta' o 'appassita"' (29-32).

2 II concetto aristotelico di mimesis è da collocarsi in un contesto preciso, cioc̀ ncll'ambito dell'atomismo ove la materia è costituita da un aggregarsi di particelle guidate da un telos in moto perpetuo verso l'ordine universale, il riposo totale. Qui, la mimesi è l'imitazione 
della realtà in atto, dunque, come scrive Cooper, si tratta di presentare e di esprimere le cose nella loro azione, di dare un senso a come le cose sono percepite, di andare oltre al senso letterale (immagine denotativa, statica) verso il senso figurato (immagine connotativa e polisemica, dinamica) (221-223). Ricoeur riprende questo concetto di mimesi e lo pone alla base delle sue teorie sul rapporto referenziale tra letteratura, realtà ed azione metaforica. Si tratta di sorpassare l'impasse della differenza tra testo fittizio e mondo reale grazie ad una nuova posizione interpretativa del lettore: la realtà è temporaneamente messa in uno stato di sospensione. Il referente del testo non è la realtà in quanto tale, bensì la proposizione di un mondo che permette di proiettare le forme possibili d'esistenza del lettore stesso. Questo referente è di secondo grado, è una denotazione metaforica - "référence dédoublée" - cioè permette la produzione di un senso nuovo, di un'ipotesi della realtà , di una diversa relazione con il mondo. In questo modo il discorso speculativo e quello letterario si combinano e creano una serie di tensioni attivate nella letteratura. Si vedano Du texte à l'action 101-117 e La métaphore vive 273-321. Scrive Singer a proposito di mimesi e di oggetto artistico: "If we discredit the correspondance theory of imitation that grew out of the critical commonplace 'art imitates life', and if like Joyce and Ricoeur, we understand the word mimesis to denote the creation of a structure for cognitive reflection equivalent to the productive capacities of nature, then we must also seek criteria for understanding the mimetic artifacts that go beyond a theory of reference" (165).

3 A proposito del funzionamento della metafora scrive Tourangeau: "Metaphors involve two systems, often drawn from different domains of experience. Despite their fundamental incompatibility, the two systems can stay married because the one serves as a model for the other. The view may sound vague (and it is vague) but it is a useful starting point, full of implications for the questions of how we know metaphors when we see them, how we interpret them, and why we prefer some metaphors to others" (18).

4 Progetto di Calvino era di scrivere cinque racconti, uno per ogni senso, da includere nel libro. Mancano quindi i racconti sul tatto e la vista. Basta però ricordare il suo romanzo Palomar e rintracciare l'importanza, tematica e strutturale, che l'autore conferisce alla vista e allo sguardo.

5 Questo racconto è apparso per la prima volta col titolo di "Sapore, sapere", in Franco Maria Ricci (giugno 1982): 63-77. Il titolo significativo indica chiaramente la tematica ripresa da Calvino in tutti i racconti sui sensi: i sensi conducono ad un tipo di conoscenza.

6 Il re in ascolto ritrova alla fine la sua vita concreta poiché si rivolge con un atto percettivo alla realtà esterna e non solo al mondo chiuso del palazzo. Egli si pone in modo indistinto di fronte all'oggetto e all'altro: "Anche tu c'eri, prima. E adesso? Non sapresti rispondere. Non sai quale di questi respiri è il tuo respiro. Non sai più ascoltare" (Sotto 89); si aziona così un meccanismo, detto da Fonzi e Sancipriano, metaforico: "Da un punto di vista psicologico quindi la metafora consiste in un atto creativo e consapevole di transfert di significato, ma tale atto è possibile solo se il soggetto è effettivamente in grado di compiere un'operazione di metaforizzazione, di porsi cioè in modo indifferenziato di fronte all'oggetto" (79).

7 Scrive Paul de Man a proposito dei concetti di lettura retorica e di semiosi illimitata di Ch. S. Peirce: "The sign is to be interpreted if we are to understand the idea it is to convey, and this is so because the sign is not the thing but a meaning derived from the thing by a process here called representation that is not simply generative, i.e. dependent on a univocal origin. The interpretation of the sign is not, for Peirce, a meaning but another sign; it is a reading, not a decodage, and this reading has, in its turn, to be interpreted into another sign, and so on ad infinitum. Peirce calls this process by means of which "one sign gives birth to another" pure rhetoric, as distinguished from pure grammar, which postulates the possibility of unproblematic, dyadic meaning and pure logic, which postulated the possibility of the universal truth of meanings" (29). 


\section{OPERE CITATE}

Calvino, Italo. Se una notte d'inverno un viaggiatore. Torino: Einaudi, 1979.

Sotto il sole giaguaro. Milano: Garzanti, 1986.

Conte, Giuseppe. La metafora barocca. Saggio sulle poetiche del seicento. Milano: Mursia, 1972.

Cooper, David E. Metaphor. Oxford: Basil Blackwell, 1986.

De Man, Paul. "Semiology and Rhetoric". Diacritics (Fall 1973): 27-33.

Eco, Umberto. "Per un'analisi componenziale dei tropi". In Retorica e poetica. Eds. D. Goldin e G. Folena. Padova: Liviana editrice, 1979: 1-8.

Semiotica e filosofia del linguaggio. Torino: Einaudi, 1984.

Fonzi, Ada e Elena Negro Sancipriano. La magia delle parole: alla riscoperta della metafora. Torino: Einaudi, 1979.

Kundera, Milan. L'incredibile leggerezza dell'essere. Trad. Antonio Barbato. Milano: Adelphi, 1985.

Ricoeur, Paul. Du texte à l'action. Paris: Seuil, 1986.

La metaphore vive. Paris: Seuil, 1975.

Singer, Alan. A Metaphoric of Fiction. Discontinuity and Discourse in the Modern Novel. Tallahassee: University Presses of Florida, 1983.

Tourangeau, Roger. "Metaphor and Cognitive Structure". In Metaphor: Problems and Perspectives. Ed. David S. Miall Sussex: The Harvester Press Ltd. 1982, 14-35. 